\title{
Schwangerschaftsabbruch: die Praxis der Spitäler und Kliniken in der Schweiz
}

\author{
Die öffentlichen und privaten Spitäler wurden gefragt, ob sie Schwangerschafts- \\ abbrüche nach der 12. Schwangerschaftswoche auch bei schwerer seelischer Not- \\ lage vornehmen und wenn ja, bis zu welcher Woche.
}

\author{
Anne-Marie Rey ${ }^{a}$, \\ André Seidenbergb \\ a Sekretariat APAC-Suisse \\ b Präsident APAC-Suisse, \\ Research Fellow Institut für \\ Hausarztmedizin und \\ Versorgungsforschung der \\ Universität Zürich IHAMZ
}

\footnotetext{
* Zusammenschluss von Fachleuten, die im Bereich ungewollte Schwangerschaft bzw. Schwangerschaftsabbruch (SA) tätig sind. Ziel ist die Qualitätssicherung bei der Durchführung des SA und die Gewährleistung des Zugangs für alle Frauen.
}

Interessenbindungen: keine

Korrespondenz:

Dr. med. André Seidenberg

Facharzt Allgemeinmedizin FMH

Weinbergstrasse 9

CH-8001 Zürich

andre.seidenberg@hin.ch

Anne-Marie Rey

Sekretariat APAC-Suisse

Grabenstrasse 21

CH-3052 Zollikofen

Tel. 0319115794

apac-suisse@bluewin.ch

wWw.

schwangerschaftsabbruch.org

\section{Einleitung}

Seit 2002 hat die Schweiz bezüglich Schwangerschaftsabbruch (SA) ein liberales Gesetz und eine vergleichsweise gut funktionierende Praxis. Aufgrund einzelner Erfahrungen und einer APAC-Suisse-Umfrage bei den schweizerischen Familienplanungsstellen [1] vom Winter 2008/2009 vermuteten wir Versorgungslücken im Besonderen bei SA nach der 12. Woche Amenorrhoe.

Die vom Parlament im März 2001 beschlossene und vom Volk im Juni 2002 mit 72\% der abgegebenen Stimmen gutgeheissene Fristenregelung erlaubt den Abbruch einer Schwangerschaft bis zur 12. Schwangerschaftswoche (SSW) auf Antrag der Frau. Danach ist gemäss StGB Art. 119, Absatz 1 ein Abbruch gestattet, «wenn er nach ärztlichem Urteil notwendig ist, damit von der schwangeren Frau die Gefahr einer schwerwiegenden körperlichen Schädigung oder einer schweren seelischen Notlage abgewendet werden kann. Die Gefahr muss umso grösser sein, je fortgeschrittener die Schwangerschaft ist.»

Diese vom Gesetz vorgesehene, zeitlich nicht beschränkte Indikation lässt einen breiten Ermessensspielraum in der Beurteilung des Einzelfalls. Im französischen Wortlaut wird das umfassende Verständnis für die Not der schwangeren Frau besonders deutlich: «danger d'une atteinte grave à l'intégrité physique ou d'un état de détresse profonde». Wir haben daher die Spitäler danach gefragt, ob sie Schwangerschaftsabbrüche nach der 12. SSW auch bei schwerer seelischer Notlage vornehmen und wenn ja, bis zu welcher SSW.

Ende März 2009 wurden die öffentlichen Spitäler und Privatkliniken sowie uns bekannte Tageskliniken in der ganzen Schweiz per E-Mail mit einem Fragebogen angeschrieben, um Informationen über ihre aktuelle Praxis des SA zu erhalten. Spitälern, die nach zweimaliger Mahnung noch nicht geantwortet hatten, wurde der Fragebogen im Oktober 2009, nach vorgängigem telefonischem Kontakt, ein viertes Mal zugeschickt. Bei Kliniken, die ausschliesslich das Belegarztsystem kennen, wurden Belegärztinnen und -ärzte direkt angeschrieben. Die Praxis einzelner Ärzte kann verschieden sein. Die Auswertung stützt sich in diesen

\section{Résultats d'une enquête de I'APAC-Suisse en 2009: Interruption de grossesse - la pratique des établissements hospitaliers en Suisse}

En dépit de sept années d'une législation et d'une pratique libérales, nous avons soupçonné certaines lacunes dans l'offre des soins médicaux pour l'interruption de grossesse (IG) en Suisse. En été 2009, une enquête fut menée auprès des hôpitaux publics et privés, y compris quelques cliniques de jour dont nous avions connaissance. Sur les 157 cliniques contactées, 113 réponses ont pu être évaluées (72\%).

93 cliniques (82\%) effectuent des IG. Au premier trimestre, les deux tiers pratiquent aussi bien la méthode chirurgicale que (dans les premières 7 ou 9 semaines) la méthode médicamenteuse par mifépristone plus misoprostol. 12 cliniques ( $13 \%$ ) offrent I'anesthésie locale pour l'intervention chirurgicale. $49 \%$ des cliniques acceptent l'indication d'un état de détresse profonde pour I'IG après le délai de 12 semaines d'aménorrhée, dans un cadre limité. L'accès à I'IG au cours du deuxième trimestre, voire au-delà, est restreint, il n'est pas fait usage sufissamment de la marge d'appréciation laissée par la loi.

Fällen auf die Antworten eines Arztes oder manchmal mehrerer Ärztinnen.

Gefragt wurde:

- Bis zu welcher SSW werden Abbrüche durchgeführt? 
- Bis zu welcher SSW werden nach der 12. Woche Abbrüche aus psychosozialer Indikation (Art. 119,1 StGB: «schwere seelische Notlage») durchgeführt?

- Welche Methoden kommen zur Anwendung?

- Dauer des Klinikaufenthaltes?

Die Auswertung des strukturierten Fragebogens erfolgte durch Auszählen mit Hilfe einer Excel-Tabelle.

\section{Resultate}

Von 157 angeschriebenen Kliniken kamen 113 (72\%) auswertbare Antworten zurück (Tab.1).

In 17 dieser 113 Spitäler und Kliniken werden keine SA durchgeführt. Es handelt sich um 5 (kleine) öffentliche Einrichtungen, die anderen sind Privatkliniken, wovon sechs konfessionelle Trägerschaften aufweisen oder durch Kaufverträge mit früheren derartigen Trägerschaften gebunden sind. Drei Privatkliniken geben an, extrem selten SA durchzuführen,

eine davon ausschliesslich bei medizinischer Indikation, aber dann meist im zweiten Trimester.

93, das heisst $82 \%$ der antwortenden Kliniken führen SA durch.

\section{Bis wann werden Schwangerschaftsabbrüche durchgeführt?}

Von diesen 93 Einrichtungen beschränken sich 22 auf Abbrüche bis zur 12. SSW (Tab. 2). Zum einen Teil sind dies Tages- und Privatkliniken, zum andern Teil kleinere öffentliche Spitäler. Die Angaben von 10 Spitälern sind diesbezüglich unvollständig. Nach der 22. Woche werden Schwangerschaften nur vereinzelt in Regional- und Privatspitälern und auch nicht in allen Kantonsspitälern abgebrochen. In 19 Spitälern wird auch nach der 24. SSW abgebrochen, nämlich in den seltenen Ausnahmefällen, wenn das Leben der Schwangeren auf dem Spiel steht (zum Beispiel bei HELLP-Syndrom) oder bei letaler fetaler Anomalie.

\section{Schwangerschaftsabbrüche wegen seelischer Notlage}

Rücklauf: Wo werden SA durchgeführt?

Total angeschriebene Kliniken

Deutsche Schweiz / GR / Oberwallis

Romandie

Tessin

Spital / Klinik inzwischen geschlossen $(2 \mathrm{~d} \mathrm{CH})$

Keine gynäkologische Abteilung ( $3 \mathrm{~d} \mathrm{CH}, 4 \mathrm{f} \mathrm{CH}$ )

Antwort ausdrücklich verweigert ( $3 \mathrm{~d} \mathrm{CH}, 1 \mathrm{fCH}$ )

Keine Antwort (17 d CH, 11 f CH, 3 TI)

Auswertbare Antworten

Davon machen keine oder praktisch keine SA

Kliniken, die SA durchführen

Kliniken, die explizit $S A>12$. SSW durchführen

Kliniken, SA >12. SSW mit psychosoz. Indikation

Von 113 Schweizer Kliniken, die auf eine Umfrage der APAC-Suisse geantwortet haben, führen 93 (82\%) Schwangerschaftsabbrüche durch. Von diesen nehmen $69 \%$ auch nach der 12. Schwangerschaftswoche Abbrüche vor, aber bloss $49 \%$ auch bei psychosozialer Indikation («schwere seelische Notlage», gemäss StGB Art. 119 Abs. 1).

\section{Tabelle 2}

Bis zu maximal welcher Schwangerschaftswoche wird in den 93 Kliniken ein SA durchgeführt?

Bis zur 12. SSW

Bis zur 15. SSW

Bis zur 18. SSW

Bis zur 22. SSW

Bis zur 24. SSW

In extremen Ausnahmefällen auch später

Unvollständige / unklare Angaben
157

105

44

8

2

7

4

31

113

20

93

64

$82 \%$

$57 \%$

$100 \%$

$69 \%$

$49 \%$
$46 \%$ der Kliniken nehmen Schwangerschaftsabbrüche auch nach der 18 . Schwangerschaftswoche vor, $20 \%$ in extrem seltenen medizinisch indizierten Ausnahmefällen auch nach der 24. Woche.
Von jenen 64 Spitälern, die nach eigenen Angaben SA nach der 12. SSW durchführen, akzeptieren 18 (28\%) eine «schwere seelische Notlage» nicht als Indikation. Das heisst, nach der 12. SSW wird eine Schwangerschaft nur abgebrochen, wenn streng somatisch-medizinische Gründe vorliegen (körperliches Risiko für die Frau, fetale Anomalie). 46 Spitäler hingegen akzeptieren schwere seelische Notlagen als Indikation zum SA, allerdings zu einem grossen Teil zeitlich sehr beschränkt, nämlich 17 Spitäler bis maximal zur 15. SSW (Tab. 3). Nur 8 Spitäler geben an, solche Indikationen auch nach der 18. SSW zu berücksichtigen.

An 33 Orten wird in solchen Fällen eine psychiatrische Begutachtung verlangt, obwohl dies vom Gesetz nicht vorgeschrieben ist. In 18 Spitälern werden sie einem klinikinternen Gremium unterbreitet. Ein Spital verlangt gar zwei ärztliche Gutachten und zwei Spitäler nehmen Rücksprache mit dem Kantonsarzt.

\section{Welche Methoden kommen zur Anwendung?}

\section{Medikamentöser Schwangerschaftsabbruch im 1. Trimester}

21 der 93 Einrichtungen, welche SA im ersten Trimester durchführen, geben an, dass sie die medikamentöse Methode mit Mifepriston (Mifegyne ${ }^{\circledR}$ ) plus Misoprostol $\left(\right.$ Cytotec $\left.^{\circledR}\right)$ für frühzeitige SA nicht anbieten. Es sind dies vor allem kleine und private Kliniken. Frauen werden für diese Behandlung in grössere Spitäler überwiesen oder in der Praxis von Belegärzten behandelt. 6 Kliniken haben die Frage nach dieser Methode nicht beantwortet.

In 50 der 66 Einrichtungen, welche die Methode anbieten, bleiben die Frauen in der Regel bis zu vier Stunden zur Beobachtung, andernorts länger. In 25 Einrichtungen haben die Frauen die Wahl, das 


\section{Tabelle 3}

SA nach der 12. SSW aus psychosozialer Indikation an den 46 Kliniken, die solche durchführen.

Bis 13. SSW

2

Bis 14. SSW

10

Bis 15. SSW

Bis 16. SSW

Bis 18. SSW

Bis 20. SSW

Bis 22. SSW

Bis 23. SSW

Keine Angabe, bis zu welcher SSW

Von 46 Kliniken, die im 2. Trimenon psychosoziale Gründe (eine «schwere seelische Notlage») für einen Schwangerschaftsabbruch akzeptieren, tun dies rund ein Viertel nur bis zur

14 . Woche und ein knappes Viertel auch noch nach der 16. Woche. Der gesetzliche Rahmen wird nicht ausgeschöpft.
In 40 Spitälern und Kliniken wird der Eingriff auch nach der 12. SSW operativ vorgenommen, meist jedoch maximal bis zur 13. bzw. 14. SSW, in 5 Spitälern bis zur 15. oder 16. Woche, in einem Spital bis zur 20. Woche.

In der Regel wird der Eingriff ambulant durchgeführt, mit einem Klinikaufenthalt von 4 bis 12 Stunden. In 14 Einrichtungen bleiben die Patientinnen normalerweise weniger als 4 Stunden. In 6 Spitälern hingegen, darunter sowohl Privatkliniken wie öffentliche Spitäler, ist eine Hospitalisation die Regel und in 4 weiteren kommt beides vor, stationär wie ambulant.

\section{Diskussion}

In allen Regionen der Schweiz haben Frauen die Möglichkeit zum SA. Es gibt jedoch Lücken in der Versorgung und Hinweise auf lokale Qualitätsmängel. Nicht überall hat die Frau eine Wahl bei der Methode und beim Prozedere. Die teilweise beschränkten Möglichkeiten für einen SA können sich insbesondere für Frauen mit knappen Ressourcen, für Jugendliche [2] und Migrantinnen [3] negativ auswirken.

Die vorliegende Umfrage bei Spitälern und Kliniken in der Schweiz ist mit einem Rücklauf von $72 \%$ hochrepräsentativ. Nicht erfasst wurden ambulante SA in Privatpraxen niedergelassener Ärztinnen und Ärzte. Dort werden SA meist nur mit der medikamentösen Methode durchgeführt. Bloss ein gutes Dutzend Frauen- und Allgemeinärztinnen und -ärzte [4] in der Schweiz führen ambulante, chirurgische SA in Privatoder Gruppenpraxen durch.

Nur gerade 11 Kliniken (12\%) akzeptieren die Indikation der «schweren seelischen Notlage» auch nach der 16. SSW. Der durch diese Formulierung gesetzlich zulässige weite Ermessensspielraum [5] wird in den meisten Institutionen nicht ausgeschöpft. Ausserdem kommt auch im frühen zweiten Trimenon oft ein restriktiveres Prozedere zur Anwendung, als das Gesetz vorschreibt: Die Frau muss sich einer psychiatrischen Begutachtung unterziehen oder es wird gar Rücksprache mit dem Kantonsarzt genommen. Laut Bundesgerichtsentscheid [6] kann die behandelnde Ärztin oder der behandelnde Arzt ohne Zweitmeinung entscheiden, ob eine schwere seelische Notlage vorliegt.

Wenn der SA nach der 12. SSW in einem Kanton abgelehnt wird, kann die Frau aus versicherungstechnischen oder administrativen Gründen von den meisten Kliniken in anderen Kantonen nicht aufgenommen werden. Hier liegt der Grund dafür, dass gemäss unserer Umfrage bei den Familienplanungsstellen und unseren Erkundigungen bei den ausländischen Kliniken jährlich um die 50 Frauen aus der Schweiz für einen SA nach dem ersten Trimenon ins Ausland reisen.

SA im ersten Trimenon werden an Privatkliniken selten mit der medikamentösen Methode durchgeführt. Mifepriston ist in der Schweiz zusammen mit Misoprostol für den SA bis zum 49. Tag der Amenorrhoe zugelassen. Hingegen hat die europäische Arzneitiziert, an einem kleinen Spital ausschliesslich diese.

\section{Saugcurettage}

48 Kliniken ist eine intravenöse Vollnarkose möglich. In 54 Kliniken kommt auch die Regionalanästhesie zum Einsatz und in 42 allenfalls eine Intubationsnarkose. Lokalanästhesie beim chirurgischen SA wird nur in 12 Einrichtungen angeboten. An 14 Orten wird neben der Saugcurettage unter
Umständen auch die konventionelle Curettage prak-

Misoprostol zu Hause einzunehmen. In 10 Kliniken erfolgt meist stationär. 16 Spitäler und Kliniken geben an, SA auch in diesen Fällen ambulant (ohne Übernachtung im Spital) vorzunehmen. 
